\title{
Performance of Jet, Missing Transverse Energy and Tau Reconstruction with ATLAS in pp Collisions at $\sqrt{s}=7 \mathrm{TeV}$
}

\author{
Ariel Schwartzman ${ }^{* \dagger}$ \\ SLAC National Accelerator Laboratory \\ E-mail: ariel.gustavo.schwartzman@cern.ch
}

\begin{abstract}
Preliminary results of jet, missing transverse energy, and tau reconstruction and calibration with the ATLAS detector from proton-proton collisions at $\sqrt{s}=7 \mathrm{TeV}$ are presented. Inputs to jet, tau, and missing $E_{T}$ reconstruction and calibration are studied and validated with data. The jet energy calibration is derived from simulated QCD dijet events and its uncertainty determined using a combination of Monte Carlo, data, and test-beam data measurements. The initial ATLAS jet energy scale is determined with an uncertainty smaller than $10 \%$ for jets with transverse momentum greater than $20 \mathrm{GeV}$.
\end{abstract}

35th International Conference of High Energy Physics

July 22-28, 2010

Paris, France

\footnotetext{
*Speaker.

${ }^{\dagger}$ On behalf of the ATLAS Collaboration
} 


\section{Introduction}

A precise understanding of jet, missing transverse energy $\left(E_{T}^{\text {miss }}\right)$, and tau reconstruction and calibration are critical for the physics program of the Large Hadron Collider. In particular, the jet energy scale is one of the dominant uncertainties in most physics analysis involving jets, such as single, di-jet and multi-jet cross sections, and beyond the Standard Model searches with jets and large $E_{T}^{\text {miss }}$ in the final state. Data collected from proton-proton (pp) collisions at $\sqrt{s}=7 \mathrm{TeV}$ with the ATLAS detector during 2010 was used to validate the inputs to jet, $E_{T}^{\text {miss }}$, and tau reconstruction and calibration and to set the first jet energy scale uncertainty.

The reconstruction and calibration of jets, taus, and $E_{T}^{\text {miss }}$ rely on the ATLAS calorimeter system, which consists of several sub-detectors covering the pseudorapidity region $|\eta|<4$.9. A highly granular Liquid Argon (LAr) electromagnetic (EM) calorimeter covers the region $|\eta|<3.2$. The hadronic calorimeter in the central $(|\eta|<1.7)$ region is made of steal and scintillating fibers, whereas in the endcaps $(1.5<|\eta|<3.2)$ LAr technology is used. The forward region extends the $\eta$ coverage up to 4.9 with electromagnetic and hadronic LAr calorimeters. The ATLAS calorimeters and the rest of the sub-detectors are described in detail elsewhere [1].

\section{Jet reconstruction}

Jets are reconstructed using the infrared-safe anti- $k_{t}$ clustering algorithm [2] with resolution parameter $R=0.6$ and 0.4 with full four-momentum recombination. ATLAS uses three different input signals to find jets: topological clusters (topo-clusters), calorimeter towers, and tracks. Topo-clusters are dynamically formed three-dimensional objects optimized to follow the shower development. They only include cells with significant signal or directly neighboring it. See [3] for details. Towers are built by projecting all calorimeter cells associated to topo-clusters into a fixed geometrical grid of size $\Delta \eta \times \Delta \phi=0.1 \times 0.1$. The use of cells belonging to topo-clusters in the definition of towers provides an effective noise-suppression for tower signals. Finally, tracks reconstructed with the inner detector coverage of $|\eta|<2.5$ provide an input signal to reconstruct jets independent of calorimeter measurements and that contains additional $z$-vertex information which makes it less sensitive to pile-up effects. A detailed validation of inputs to jet reconstruction and calibration is provided in [3].

\section{Jet energy calibration}

Reconstructed calorimeter jets are calibrated at the electromagnetic (EM) scale, established using test-beam measurements from electrons in the electromagnetic and hadronic calorimeters. Measurements of single particle response in data [4] and test-beam [5] [6] indicate that the detector simulation describes the EM scale of hadrons within 5\%. Jets reconstructed at the EM scale need to be corrected for the lower ATLAS calorimeter response to hadrons (non-compensation) and for other instrumental effects such as energy losses in inactive regions of the detector (dead material), particles not fully contained in the calorimeter (leakage) or that fall out the jet boundaries due to the bending of charged particles in the magnetic field, and inefficiencies in the clustering and jet reconstruction. The jet energy scale is derived as a global function of $\eta$ and $p_{T}^{j e t}$ that corrects the 

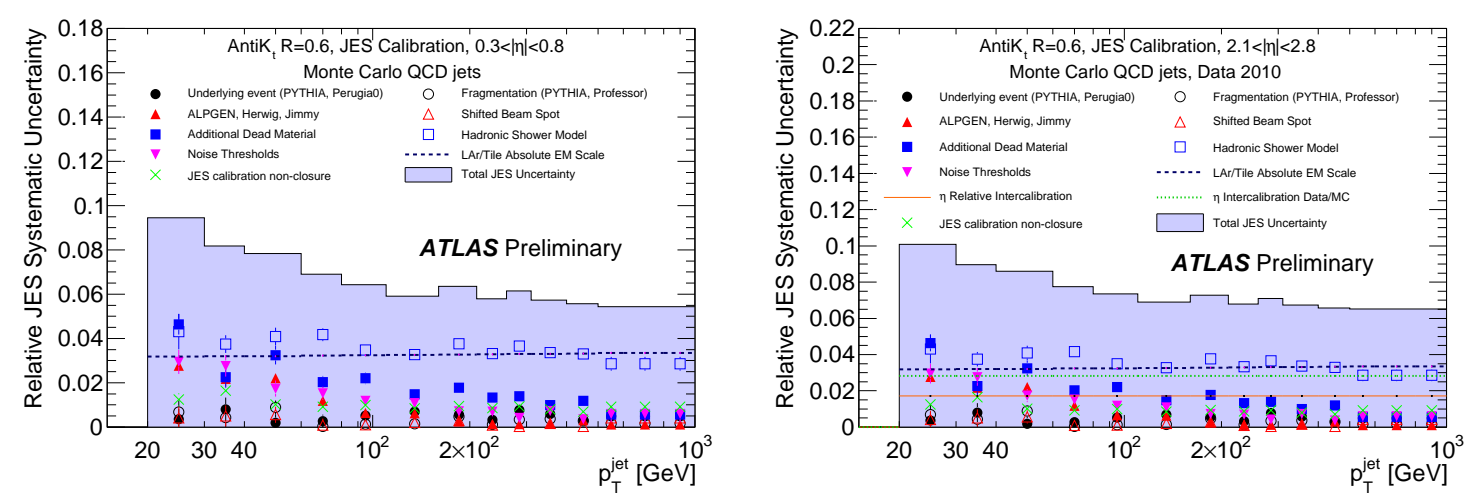

Figure 1: Relative jet energy scale systematic uncertainty as a function of $p_{T}^{\text {jet }}$ for anti- $k_{t} \mathrm{R}=0.6$ jets with topo-cluster constituents in the pseudorapidity region $0.3<|\eta|<0.8$. The total uncertainty is shown as the solid light blue area. The individual sources are also shown, with statistical errors if applicable.

energy and momentum of jets using as a reference the corresponding Monte Carlo truth jets in QCD dijet events. The jet energy scale uncertainty has been determined using a combination of single pion test-beam measurements and Monte Carlo samples generated with different conditions with respect to the nominal sample used to derive the jet energy scale. It accounts for uncertainties on the absolute electromagnetic scale, the material budget of the calorimeter, the description of the electronic noise, the hadronic shower model used in the detector simulation, the fragmentation and underlying event models used in the Monte Carlo generators, and differences in the beam spot between data and simulation. The jet energy scale uncertainty is determined for the barrel region and then extrapolated to the endcap and forward region using dijet inter-calibration measurements in data [7]. The jet energy uncertainty due to in-time pile-up was estimated directly from data, using measurements of tower energy density as a function of the number of additional multiple interactions. Figure 1 shows the relative jet energy scale uncertainty as a function of $p_{T}^{\text {jet }}$ in the central and endcap regions. The overall uncertainty is smaller than $7 \%$ for $p_{T}^{\text {jet }}>60 \mathrm{GeV}$ [8]. The jet energy resolution, measured in-situ using the dijet balance and bisector methods, agrees within $14 \%$ with the simulation for jets with $|y|<2.8$ [9]. ATLAS has also validated three advanced calibration schemes that significantly improve the jet energy resolution. The Global Sequential Calibration (GSC) uses jet-by-jet information about the longitudinal and transverse properties of the jet. The Global Cell Weighting (GCW) uses cell weights based on the cell energy density to compensate for the different calorimeter response to hadronic (low energy density) and electromagnetic depositions. The Local Cluster Weighting (LCW) uses properties of topo-clusters to calibrate them individually. The detailed validation of all calibration schemes is described in [3]

\section{Missing transverse energy and tau reconstruction and performance}

The $E_{T}^{\text {miss }}$ is reconstructed from cells belonging to topo-clusters and from reconstructed muons removing the contribution from the energy deposition in the calorimeter cells. Cells in topo-clusters are calibrated using the LCW calibration scheme described in 3. Figure 2 shows an excellent agreement between data and Monte Carlo in the distribution of the $x$ and $y$ components of the $E_{T}^{\text {miss }}$ in minimum bias events. The $E_{T}^{\text {miss }}$ resolution is also very well described by the simulation and 

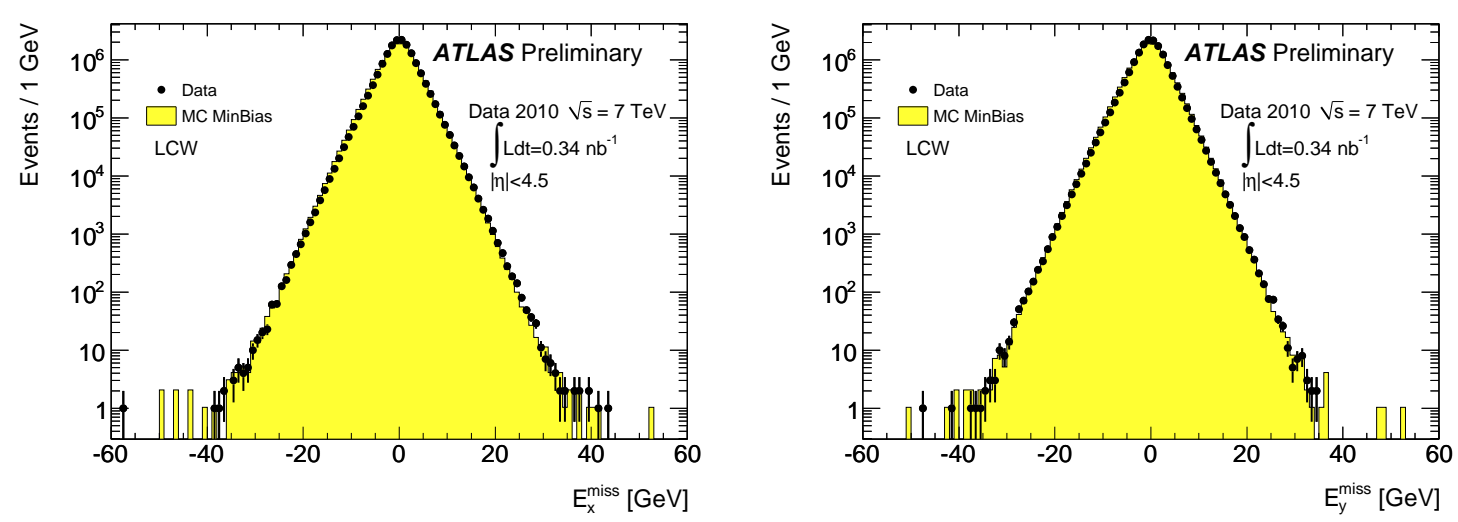

Figure 2: Distributions of the $x$ (left) and $y$ (right) components of the transverse missing $E_{T}$ in minimum bias data and Monte Carlo.

measured to be $0.51 \sqrt{\sum E_{T}} \mathrm{GeV}$. The $E_{T}^{\text {miss }}$ reconstruction and calibration has also been validated in $W \rightarrow l v$ events [10].

Hadronic taus are reconstructed using a combination of calorimeter and tracking information that allow to separate the tau signal from the jet background. The validation of the inputs to tau reconstruction and calibration is described in [11]. Very good agreement is observed between data and Monte Carlo.

\section{Conclusions}

ATLAS has developed several jet, tau, and $E_{T}^{\text {miss }}$ reconstruction and calibration methods, with different sensitivities to systematic effects. Inputs to jet, tau, and $E_{T}^{\text {miss }}$ reconstruction and calibration were validated with collision data and found to be well described by the simulation within $10 \%$. The first ATLAS jet energy scale has been determined with an uncertainty smaller than $7 \%$ for jets with $p_{T}>60 \mathrm{GeV}$.

\section{References}

[1] The ATLAS Collaboration, JINST 3, S08003 (2008)

[2] M. Cacciari, G. P. Salam and G. Soyez, JHEP 0804, 063 (2008)

[3] The ATLAS Collaboration, ATLAS-CONF-2010-053

[4] The ATLAS Collaboration, ATLAS-CONF-2010-052

[5] The ATLAS Collaboration, Nucl. Instrum. Meth. A614 (2010) 400-432

[6] The ATLAS Collaboration, ATL-CAL-PUB-2010-001

[7] The ATLAS Collaboration, ATLAS-CONF-2010-055

[8] The ATLAS Collaboration, ATLAS-CONF-2010-058

[9] The ATLAS Collaboration, ATLAS-CONF-2010-058

[10] The ATLAS Collaboration, ATLAS-CONF-2010-039

[11] The ATLAS Collaboration, ATLAS-CONF-2010-059 